\title{
A new landscape for distributed and parallel data management
}

\author{
Amit Sheth
}

Published online: 4 April 2012

(C) Springer Science+Business Media, LLC 2012

The inaugural issue of Distributed and Parallel Databases (DAPD) appeared in 1993. It was an outcome of this journal's founding editor Dr. Ahmed Elmagarmid's vision. In the first editorial, he noted the need to provide a high quality forum to "publish original results related to databases and systems that use distributed or highly parallel platforms, parallel algorithms, issues resulting from parallelism and distribution of application and data." DAPD indeed started at the right time, and its relevance grew as database technologies were increasingly deployed in large-scale distributed infrastructures and used parallelism and distribution to improve scale and performance. DAPD faithfully served this mission and made a space among database journals with its focus. Ahmed was early in identifying the topics that grew in importance, such as interoperability in multidatabase systems, object-oriented databases, knowledge bases, and managing biological, spatial, scientific and statistical data.

Ahmed carried the ball as the Editor-in-Chief (EIC) for over 15 years, and then as a co-EIC once he called upon me to join him four years ago. A couple of years ago, Ahmed took up a significant leadership position at the Qatar Foundation, and along with that, significant responsibilities. So it is understandable that Ahmed has decided to pass on the DAPD leadership role to others. I am confident that the database community will continue to receive the benefits of Ahmed's leadership and service, but DAPD owes its immense gratitude for all that he has done for this journal.

This occasion also affords me a privilege to welcome one of the best known database researchers, Divyakant (Divy) Agrawal. Divy has been on the faculty of Computer Science Department at the University of California at Santa Barbara since 1987 where he currently serves as a Professor of Computer Science. He is an active

\footnotetext{
A. Sheth $(\bowtie)$

Kno.e.sis Center, Wright State University, Dayton, OH 43435, USA

e-mail: amit@knoesis.org
} 
member of the Database and Distributed Systems research community and his contributions have been recently recognized. In 2012, he has been inducted as a Fellow in both the IEEE and the ACM. Divy also served as an Associate Editor on the Editorial Board of DAPD since its inception until 2009. Although Ahmed and I had started to ensure that DAPD remains highly relevant to database researchers, Divy and I will proceed to expedite the positioning and broadening of its scope, so that they are in line with the growth and success of the World Wide Web that is now ubiquitous and pervasive with the spread of mobile devices and emergence of Internet of Things, new computational themes, such as cloud computing and big data, and the continued growth of increasing variety of data including social and sensor data, each presenting us with unique data management challenges and new opportunities. Let me review the key recent factors and trends.

Data is generated everywhere: We have surpassed 5 billion mobile phones and 40 billion mobile sensors. An increasing number of these devices are generating data that can be accessed over the Internet and the Web. With the emergence of Internet of Things (IoT), everyday objects are becoming data sources, massively increasing the amount of available data.

Data is more heterogeneous: In the 1980s and 1990s, data was primarily generated and used in enterprises and was structured. Last decade saw a massive increase in unstructured data. With Web 2.0, we have witnessed an increasing amount of Web accessible scientific and engineering data, user generated content, and streaming data. Now, we see rapid growth in sensors and Internet of Things. Often applications need to deal with a variety of data-structured or unstructured text, multimedia content, multimodal data, static data, dynamic or streaming data and so on. Thus, while we continue to make progress in addressing some of the interoperability and integration challenges, we are faced with even more diverse types of data.

Data is more complex and nuanced: Early IT systems used to have preplanned data organization, usually with a schema that would describe the organization of data. The first challenge is that Increasingly, we are faced with data that is schema-less, i.e. the organization or structure of data would continue to change. The second challenge we face is that, for a given event or activity, we often have data of different modality, giving complementary or overlapping perspective. For example, a traffic event would be recorded and reported by machine sensors, as well as citizen sensors (e.g., by a tweet). The third challenge we face is that, unlike data in the past that primarily captured factual information or observations, data presented by a human is often highly nuanced and complex. A tweet, for example, can have spatial, temporal, thematic, people (user), network and content (including sentiment, emotion, provenance, etc.) aspects, that can be captured by different facets of metadata. Increasingly metadata is more complex, and the ratio of metadata to data continue to go up. In the face of these challenges, we have not only improved our ability to connect the data-informationknowledge dimension, but also enhanced our ability to use communicated or other forms of background knowledge (e.g., Wikipedia or Linked Open Data) to increase our ability to understand or associate meaning to data. Semantic Web technologies have provided richer representational and reasoning frameworks to deal with this kind of complex data.

Data sizes are massive: A couple of years ago, we surpassed the capacity to store all the data that is created. And just in two years, we will create more data than $90 \%$ 
of the data that exists today. So collection, storage, querying and above all, analysis of any relevant fragment of the data-what is now called Big Data-is a significant opportunity and a challenge. Businesses are increasingly looking at this Big Data as a source for business intelligence and competitive advantage, while advances in science and engineering increasingly relay in the ability to collect, integrate and analyze massive amount of heterogeneous data.

New architectures for data processing are emerging: The last decade saw the rise of multicore architectures offering newer opportunities in parallel processing and innovations in voluntary computing. We now have the ability to distribute data processing across clients and clouds - with challenges of transferring large amounts of data to and from clouds. Innovative research in this area has focused on moving code to data rather than moving data to code, and involving a large number of consumer devices in the computing ecosystem. Another important area is the emerging, highly available but weakly consistent data base systems. These systems are ideal to store the massive data being generated but bring new challenges due to their weak consistency models.

Along with most of the above data management issues, we face challenges associated with business models and monetization, user engagement, autonomy, ownership, provenance, access control, trust, privacy, and security.

Our initial steps to start addressing the aforementioned challenges and opportunities are reflected in the special issues that we have commissioned, which include:

1. Ranking in Databases-Kaushik Chakrabarti

2. Big Data-Jignesh Patel

3. Data Intensive e-Science-Judy Qiu, Dennis Gannon

4. Mobile Databases-Mohamed Mokbel, Dipanjan Chakraborty, Vana Kalogeraki

5. Security-Elena Ferrari, Murat Kantarcioglu

This is matched by some changes in our Editorial Board. We thank a number of stalwarts that have helped us for many years, including: Hector Garcia-Molina, C. Mohan, M. Tamer Ozsu, Raghu Ramakrishnan, Marek Rusinkiewicz, Avi Silberschatz, Jennifer Widom, Kam-Fai Wong, Clement Yu and Aidong Zhang. At the same time, we are delighted to bring some raising stars and some established leaders on board, including, Saurav Bhomick, Rajkumar Buyaa, Lei Chen, Yi Chen, Bin Cui, Anwitaman Datta, Reynold Cheng, Chen Li, and Haixun Whang. Our plan is to have more regular update to the editorial board to give us agility in constantly refocusing on that is emergent and important in data management. The above steps, we hope will make DAPD even more relevant and important part of the database community in the coming years.

Amit Sheth

March 17, 2012 